ARQGA / 914

\title{
FREQÜÊNCIA DE PÓLIPOS EM DOENTES OPERADOS DE CÂNCER COLORRETAL
}

\author{
Beatriz Pinho ROCHA*, Ângelo Paulo FERRARI** e Nora Manoukian FORONES**
}

RESUMO - Introdução - Estudos epidemiológicos e gênicos mostram que os pólipos colônicos do tipo adenoma são lesões pré-neoplásicas. O seguimento de um paciente com câncer colorretal visa principalmente o diagnóstico e retirada de pólipos adenomatosos. Objetivo - Estudar a freqüencia do aparecimento de pólipos adenomatosos em doentes com câncer colorretal em acompanhamento clínico após ressecção tumoral. Casuística e Métodos - Foi estudada, retrospectivamente, a freqüencia de pólipos do tipo adenoma em 68 pacientes com idade média de 59 anos, submetidos a cirurgia curativa para tratamento de câncer colorretal e a colonoscopia de controle. Resultados - A incidência de pólipos adenomatosos foi de 18\%, sendo maior acima de 45 anos (20\%) e no cólon esquerdo (38\%). Em relação ao tipo histológico, 61\% eram tubulares, 22\% vilosos e 17\% túbulo-vilosos. Discussão - Assim como em outros relatos da literatura, a incidência de pólipos foi superior em indivíduos com mais de 45 anos, sendo em mais da metade do tipo tubular. A freqüencia de pólipos foi maior nos dois primeiros anos de acompanhamento.

DESCRITORES - Pólipos do cólon. Neoplasias colorretais, cirurgia.

\section{INTRODUÇÃo}

O câncer de cólon e reto responde por $15 \%$ das neoplasias malignas nos Estados Unidos ${ }^{(1)}$. No Brasil, estima-se que 25000 novos casos foram diagnosticados em 1995 e que 9000 pessoas morreram desta patologia.

Os pólipos ocorrem com grande freqüência (16 a 41\%) no cólon e reto após os 50 anos. Os pólipos do tipo adenoma poderão sofrer transformação maligna; pacientes com adenoma têm duas a três vezes maior risco de desenvolver câncer ${ }^{(5)}$. Os adenomas podem ser classificados histologicamente em viloso (consiste em glândulas da lâmina própria com formação papilar que se estendem do epitélio até a camada muscular da mucosa), tubular (composto por glândulas compactadas na lâmina própria) e túbulo-viloso (tem características dos dois tipos). O pólipo viloso tem maior chance de malignização em relação ao tubular ${ }^{(8)}$. O pólipo do tipo hiperplásico não sofre transformação maligna e, portanto, não é uma lesão pré-neoplásica.

A colonoscopia no acompanhamento de doentes submetidos a ressecção de câncer colorretal, tem por finalidade diagnosticar e ressecar pólipos, assim como detectar novos cânceres $^{(9,10,11)}$. No setor de Oncologia do Serviço de Gastroenterologia do Hospital São Paulo, o seguimento dos pacientes tratados cirurgicamente é feito nos primeiros dois anos, a cada três meses e, posteriormente, a cada seis meses até completar cinco anos. Os exames realizados rotineiramente são:

* $\quad$ Aluna de Graduação da Universidade Federal de São Paulo, Escola Paulista de Medicina (UNIFESP-EPM). Bolsista da CAPES.

** Professor Adjunto. Setor de Oncologia - Disciplina de Gastroenterologia da UNIFESP-EPM.

Endereço para correspondência: Dra. Nora Manoukian Forones - Rua Botucatu, 740 - 04023-900 - São Paulo, SP. e-mail: nora@gastro.epm.br 
avaliação clínica, dosagem do CEA e ultrasom abdominal. Uma vez ao ano o doente deverá realizar colonoscopia e tomografia computadorizada do abdome. O seguimento de um paciente com câncer colorretal deve ser intensivo pois a recurrência ocorre em cerca de $30 \%$ dos pacientes durante os primeiros dois anos de acompanhamento pósoperatório $^{(2,3)}$.

O objetivo deste estudo foi comparar a freqüência do aparecimento de pólipos adenomatosos (considerados lesões préneoplásicas) em doentes em acompanhamento clínico após ressecção do câncer colorretal.

\section{CASUÍSTICA E MÉTODOS}

Através de estudo retrospectivo, foram estudados 68 pacientes com idade média de 59 anos (variando de 32 a 85 anos), entre janeiro de 1989 e janeiro de 1997, submetidos a ressecção cirúrgica de câncer colorretal. Os pacientes foram distribuídos de acordo com o sexo e idade (Tabela 1). Cada indivíduo foi acompanhado, em média, por 31 meses (12 a 72 meses) e submetido a pelo menos uma colonoscopia pós-cirúrgica. Foram analisadas ao todo 98 colonoscopias. Excluíram-se do estudo doentes com tumor irressecável e doentes com recidiva tumoral.

Todos os pólipos encontrados durante a colonoscopia foram removidos e enviados para estudo anatomopatológico. Foram

Tabela 1 - Distribuição dos pacientes operados e acompanhados, por sexo e idade

\begin{tabular}{lcc}
\hline & & $\mathbf{n}(\mathbf{\%})$ \\
\hline Sexo & $\mathrm{F}$ & $39(57,3)$ \\
& $\mathrm{M}$ & $29(42,7)$ \\
Idade média & Anos & 59 \\
\hline F: feminino & M: masculino &
\end{tabular}

F: feminino M: masculino excluídos pólipos hiperplásicos e processos inflamatórios, sendo estudada apenas a freqüência de pólipos do tipo adenoma.

Em alguns indivíduos foram encontrados mais de um pólipo durante o exame colonoscópico. Os resultados foram analisados pelo número de colonoscopias positivas para pólipos, independente do número destes.

\section{RESULTADOS}

A freqüência de pólipos do tipo adenoma encontrados nos doentes submetidos anteriormente a ressecção de câncer colorretal foi de $18 \%$. Observou-se maior freqüência de pólipos no sexo feminino (64\%) e nos pacientes acima de 45 anos (Tabela 2).

Foram encontradas 18 colonoscopias positivas, correspondendo a 14 pacientes. No grupo com mais de 45 anos, a freqüência de pólipos foi de $20 \%$ (Tabela 2). Cinqüenta por cento dos pólipos adenomatosos foram observados nos dois primeiros anos de acompanhamento (Figura 1).

Quanto à localização, 55\% dos pólipos adenomatosos foram encontrados no cólon

Tabela 2 - Sexo, idade e características dos doentes com pólipos

\begin{tabular}{llr}
\hline & & $\mathrm{n}(\%)$ \\
\hline Sexo & $\mathrm{F}$ & $9(64)$ \\
\hline Idade & $\mathrm{M}$ & $5(36)$ \\
& $<45$ anos & $1(7)$ \\
\hline Número de pólipos & $>45$ anos & $13(93)$ \\
\hline Número de colonoscopias positivas & & 18 \\
\hline Localização: & Cólon ascendente & $18(21)$ \\
& Cólon descendente & $10(55)$ \\
& Reto & $6(33)$ \\
Tipo histológico: & Tubular & $2(12)$ \\
& Viloso & $11(61)$ \\
& Túbulo-viloso & $3(22)$ \\
& & $3(17)$ \\
\hline
\end{tabular}

F: feminino M: masculino ascendente e $45 \%$ em reto e cólon descendente (Tabela 2). Consideramos como cólon ascendente: cólon direito e transverso e como cólon descendente: cólon esquerdo e sigmóide

Histologicamente, $61 \%$ dos pólipos adenomatosos eram tubulares, $22 \%$ vilosos e 17\% túbulo-vilosos.

\section{DISCUSSÃO}

Observou-se maior freqüência de pólipos adenomatosos nos pacientes acima de 45 anos. O percentual de pólipos em doentes com ressecção de câncer colorretal encontrado na presente série (18\%) foi menor que o encontrado em outros estudos, que variam entre 25 e $50 \%{ }^{(4,7)}$. Isto pode ser explicado pelo acompanhamento mais prolongado dos pacientes nesta casuística, ao contrário dos outros trabalhos em que os resultados são relativos aos dois primeiros anos de pósoperatório. Neste período, observaram-se que $25 \%$ dos doentes acompanhados desenvolveram pólipos adenomatosos, correspondendo a $50 \%$ dos doentes que desenvolveram pólipos.

Encontrou-se uma distribuição inversa na localização dos pólipos adenomatosos 




Figura 1 - Freqüência de aparecimento de pólipos durante acompanhamento pós-operatório quando comparada ao percentual de pólipos descritos na população geral. A freqüência maior de pólipos no cólon ascendente devese ao fato de que a maioria dos doentes com câncer colorretal foi submetida a hemicolectomia esquerda prévia, uma vez que o câncer colorretal ocorre com freqüência maior nesta localização ${ }^{(6)}$.

Conclui-se que a freqüência de pólipos adenomatosos em doentes submetidos a ressecção de câncer colorretal é de $18 \%$, sendo maior no cólon ascendente e nos primeiros dois anos de acompanhamento, reforçando a necessidade de se realizar endoscopia neste período.

Rocha BP, Ferrari AP, Forones NM. Frequency of adenomatous polyps after surgical resection of colorectal cancer. Arq Gastroenterol 2000;37(1):31-4.

ABSTRACT - Introduction - Epidemiologic and molecular biologic studies have already demonstrated that adenomatous colonic polyps are precancerous diseases. The main indication of the colonoscopy in the surveillance of colorectal cancer treated patients is the diagnosis and resection of adenomatous polyps. Aim - To study the frequency of adenomatous polyps after surgically resection of colorectal cancer. Material and Methods - Sixty eight patients, mean age 59 years old, with total resection of colorectal cancer, submitted to various colonoscopies during the follow up were studied retrospectively. The histological type and the characteristics of the polyp were described. Results - The frequency of polyps was 18\%, being higher in the patients with more than 45 years (20\%). The site of the polyps was in the left colon in $38 \%$ of the patients with cancer. The histological type of adenomas was tubular in $61 \%$, villous in $22 \%$ and mixed in $17 \%$. Discussion - As described by other authors, the incidence of polyps were higher after 45 years old and more than a half of them were tubular. The frequency of polyps was higher in the first two years of follow up.

HEADINGS - Colonic polyps. Colorectal neoplasms, surgery.

\section{REFERÊNCIAS BIBLIOGRÁFICAS}

1. Austoker J. Screening for colorectal cancer. Br Med J 1994;309:382-6.

2. Chapman I. Adenomatous polyps of large intestine: incidence and distribuition. Ann Surg 1963;157:223.

3. Gil JZ, Forones NM. IV: Intestinos. In: Forones NM, editor. Manual de diagnóstico e tratamento de doenças do aparelho digestivo. São Paulo: Ribeiro Artes Gráficas; 1996. p. 34-40.
4. Juhl G, Larson GM, Mullins R. Six-year of annual colonoscopy after resection or colorectal cancer. World J Surg, 1990;14:255-61.

5. Kronborg O, Haje E. The remaining colon after radical surgery for colorectal cancer. Dis Colon Rectum, 1983;26:172-6.

6. Morson BC. Genesis of colorectal cancer. Clin Gastroenterol 1976;5:505.

7. Morson BC, Bussey HJR. Magnitude of risk for cancer in patients with colorectal adenomas. Br J Surg 1985;72:S23-4. 
Rocha BP, Ferrari AP, Forones NM. Freqüência de pólipos em doentes operados de câncer colorretal

8. Neugut AI, Lautenbach E. Incidence of adenomas after curative resection for colorectal cancer. Am J Gastroenterol 1996;91:2096-8.

9. Rex DK. Colonoscopy: a review of its yield for cancers and adenomas by indication. Am J Gastroenterol 1995;90:353-65.

10. Rex DK. Surveillance colonoscopy after resection of colorectal polyps and cancer. ASGE 1998;6:1-4.
11. Winawer SJ, Zauber AG, Ho MN. Prevention of colorectal cancer by colonoscopyc polipectomy. N Engl J Med 1993;329:1977-81.

Recebido para publicação em 20/1/1999. Aprovado para publicação em 21/2/2000. 